\title{
ORIGINAL RESEARCH \\ Analysis of Intra-Aneurysmal Flow for Cerebral Aneurysms with Cerebral Angiography
}

L.-D. Jou

M.E. Mawad

BACKGROUND AND PURPOSE: Hemodynamics is an important factor in the development and rupture of cerebral aneurysms. Current techniques for measuring blood flow in cerebral aneurysms suffer from various limitations and have not been able to address our clinical needs. A new technique has been developed for effective evaluation of intra-aneurysmal flow based on high-frame-rate cerebral angiography, especially for flow-diverters.

MATERIALS AND METHODS: Six patients with 7 unruptured ICA aneurysms were imaged with a specially designed DSA protocol (a 3D DSA and a 2D DSA acquired at 30 frames/s, with a 2-mL/s contrast injection rate). Images of these cases were analyzed to determine the intra-aneurysmal flow based on the newly developed technique. Patient-specific aneurysm models were used for CFD calculation, and intra-aneurysmal flow rates were computed numerically. The intra-aneurysmal flow rates from the 2 methods were then compared.

RESULTS: There is a linear relationship between intra-aneurysmal flow ratios obtained from highframe-rate cerebral angiography and CFD calculation $(R=0.99)$. A high frame rate (30 frames/s) provides a better estimate of intra-aneurysmal flow than low frame rates $(7.5$ frames $/ \mathrm{s}$ and 15 frames/s)

CONCLUSIONS: The CFD calculation validates the estimate of intra-aneurysmal hemodynamics from cerebral angiography. The linear relationship obtained by using these 2 techniques can be used for real-time assessment of intra-aneurysmal hemodynamics for cerebral aneurysms.

ABBREVIATIONS: CA = cerebral angiography; CFD = computational fluid dynamics; PC-MRA $=$ phase-contrast MR angiography

H

emodynamics is regarded as an importance factor in the development and rupture of cerebral aneurysms. ${ }^{1-3}$ Various methods have been used for analysis of hemodynamics in cerebral aneurysms. ${ }^{4}$ Computational tools, such as CFD, have been used for detailed wall shear stress and flow pattern analyses, ${ }^{5,6}$ but these tools often require lengthy calculation and offer no real-time hemodynamic assessment. These calculations are also constrained by various assumptions that are regarded as limitations of numeric models. PC-MRA can measure velocity field in vivo, ${ }^{7,8}$ but it requires approximately 30 minutes for 16 time points in a cardiac cycle and is still not widely available.

Cerebral angiography remains the dominant technique for aneurysm diagnosis and has been used to quantify the hemodynamics in cerebral aneurysms. ${ }^{9-11}$ Injection of contrast medium provides the basis for clinical diagnosis, and the wash-in and washout of contrast medium offers a convenient way to observe the dynamic behavior of contrast medium. The time course of the signal at an aneurysm is often fitted into a mathematic formula to determine contributions from various flow components (convention, diffusion, or mean transit time). These approaches give indirect measures of blood flow and contrast mixing, and can potentially evaluate the effect of flow diversion. ${ }^{9,10}$ All these approaches demand an unobstructed view of the aneurysm under investigation. Considering that

Received March 1, 2011; accepted after revision January 5, 2012

From the Department of Radiology, Baylor College of Medicine, Houston, Texas.

Please address correspondence to Liang-Der Jou, Department of Radiology, Baylor College of Medicine, One Baylor Plaza, Houston, Texas 77030; e-mail: jou@bcm.tmc.edu

$\equiv$ Indicates article with supplemental on-line appendix.

http://dx.doi.org/10.3174/ajnr.A3057 most aneurysms are surrounded by branching vessels, this requirement may prohibit many clinical applications. In addition, evaluation of intra-aneurysmal flow after coil embolization would be unlikely because the aneurysm is no longer visible on the DSA images.

A new technique for examining intra-aneurysmal flow that does not require an unobstructed view of the aneurysm has been developed. With a controlled injection of contrast agent at low speed, this technique generates images in which the signal intensity is proportional to the attenuation or contrast concentration. Temporal changes of contrast concentration then reflect the pulsatility of blood flow and intra-aneurysmal flow, and the difference between signals proximal and distal to an aneurysm represents the level of intra-aneurysmal flow ratio. This flow ratio is an important indication of the amount of blood flow entering an aneurysm. This new technique permits an evaluation of flow diversion for cerebral aneurysms, even in cases of coil embolization, but the main purpose of this technique is for aneurysms treated with flow-diverters. In this study, our technique is tested on a small number of patients with aneurysm and the results are compared with CFD calculations.

\section{Materials and Methods}

\section{Cerebral Angiography}

Six patients with 7 unruptured ICA aneurysms were studied under a protocol approved by our institution. Three patients were treated by flow-diverters (Pipeline; ev3, Irvine, California) within the same week, and the remaining patients were treated within the same month as controls. These aneurysms ranged from $7 \mathrm{~mm}$ to $31 \mathrm{~mm}$, and the average size was $17 \mathrm{~mm}$. Each patient received 3D DSA and 2D DSA 


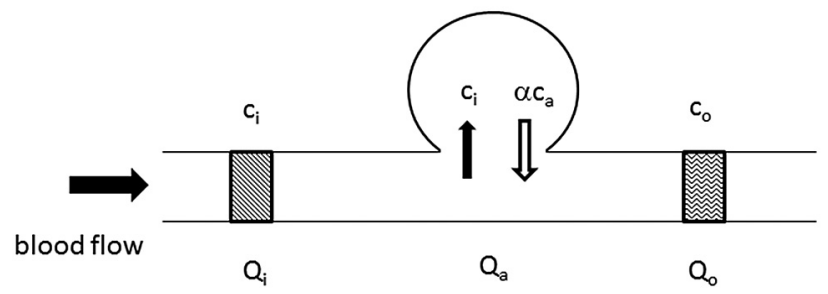

Fig 1. A sketch of balance of contrast medium transport for our model and 2 ROls, 1 proximal and 1 distal to the aneurysm. Blood enters the aneurysm with a contrast concentration $c_{i}$, but leaves with a contrast concentration of $\alpha c_{a}$. Because of heterogeneous distribution of contrast medium within an aneurysm, $\alpha \neq 1$.

on an Axiom Artis dBA (Siemens, Erlangen, Germany) as part of our clinical protocol. In 2D DSA, nondiluted contrast medium (Omnipaque 300; GE Healthcare, Piscataway, New Jersey) was injected through a $6 \mathrm{~F}$ catheter by a mechanical injector (Liebel-Flarsheim, Cincinnati, Ohio) into the ICA at $2 \mathrm{~mL} / \mathrm{s}$ for 4 seconds and the $\mathrm{x}$-ray images were acquired at $30 \mathrm{frames} / \mathrm{s}$. The tip of the catheter was at least $8 \mathrm{~cm}$ away from the aneurysm to allow for sufficient mixing of the contrast and blood. For the 2D DSA, each image was subtracted logarithmically by the mask image. In addition, a 3D DSA was acquired before the 2D DSA, and the 3D DSA permitted the planning of an optimal projection view for the subsequent 2D DSA. The 3D DSA also provided size measurements of the parent artery and aneurysm that were later used for signal processing.

Two ROIs at the ICA were selected on the 2D DSA; 1 was proximal to the aneurysm and the other was distal to the aneurysm. Both ROIs were rectangular in shape and included entire cross-sections of the ICA, as shown in Fig 1. These ROIs were within a 2-cm distance from the aneurysm; however, the distance between the region of interest and aneurysm was not a constant for all cases because of the differences in individual aneurysm morphology and projection used. Each region of interest from the 2D DSA was projected back onto the $3 \mathrm{D}$ DSA after coregistration to determine the volume of the region of interest. The volume was then used to normalize the total signal at each region of interest (see On-line Appendix for additional explanation). Another region of interest that included the entire aneurysm was selected, and the aneurysm signal was normalized by the aneurysm volume. After the normalization, each region of interest was represented by

$$
c=\frac{1}{V_{\mathrm{ROI}}} \sum_{\mathrm{ROI}} \ln \left(\frac{I}{I_{m}}\right)
$$

where $I$ was the signal and $I_{m}$ was the mask image signal. $V_{\mathrm{ROI}}$ was the volume associated with the enclosed region of interest on the 2D DSA. Because the signal $I$ was lower than the mask image signal, $c$ was negative, but its magnitude would be proportional to the local attenuation that was a function of contrast concentration. Note that $c$ had a dimension of $1 / \mathrm{m}$ and it was possible to convert the signal intensity into the Hounsfield units.

\section{Image Analysis}

Based on Fig 1, the balance of contrast medium could be written as

$$
Q_{i} c_{i}-Q_{a} c_{i}+\alpha Q_{a} c_{a}=Q_{i} c_{o},
$$

where $Q$ and $c$ were for the flow rate and concentration of contrast medium. The subscripts $i, o$, and $a$ were for the proximal region of interest, distal region of interest, and aneurysm, respectively. $Q_{i} c_{i}$ represents the contrast medium coming into the system, and $Q_{i} c_{o}$ represents the amount of contrast medium leaving the system. The amount of fluid entering the aneurysm and leaving the aneurysm would be the same, which was the intra-aneurysmal flow $Q_{a}$, but the concentration entering the aneurysm was $c_{i}$ and the concentration leaving the aneurysm was proportional to $c_{a}$, the aneurysmal concentration. This equation could be simplified as

$$
(1-f) c_{i}+\alpha f c_{a}=c_{o}
$$

where $f=Q_{a} / Q_{i}$ is the ratio of the intra-aneurysmal flow to the arterial flow rate in the parent artery. Because $c_{i}, c_{o}$, and $c_{a}$ represent the temporal history of the concentration, they could be treated as vectors in the $n$-dimensional space, where $n$ is the number of sampling points. Suppose the base vectors in the null space of $c_{a}$ were $v_{k}(k=1, \ldots, n-1)$, then we could sum up the product of the previous equation with all the base vectors, and the resulting equation would be ${ }^{12}$

$$
(1-f) \sum_{k} v_{k}\left(v_{k} \cdot c_{i}\right)=\sum_{k} v_{k}\left(v_{k} \cdot c_{a}\right)
$$

Note that $v_{k} \cdot c_{a}=0$ by the definition of a null space. With the time curves $\left(c_{i}\right.$ and $c_{o}$ ) measured from the ROIs, it would be easy to determine the intra-aneurysmal flow ratio $(f)$ as

$$
f=1-\frac{\left|\sum_{k} v_{k}\left(v_{k} \cdot c_{o}\right)\right|}{\left|\sum_{k} v_{k}\left(v_{k} \cdot c_{i}\right)\right|} .
$$

This ratio $(f)$ will be compared with the intra-aneurysmal flow ratio from CFD calculations later. Based on this formula, the difference between $c_{i}$ and $c_{o}$ determines the intra-aneurysmal flow rate. A close match between $c_{i}$ and $c_{o}$ gives a low intra-aneurysmal flow. The use of $c_{a}$ is to find the base vectors of the null space, unlike in previous studies, where all the aneurysmal dynamics depended only on the behavior of $c_{a}$. For a coiled aneurysm, the null space then was the entire vector space.

\section{Numeric Simulation}

The 3D DSA images were used to reconstruct patient-specific aneurysm models for flow simulations. A flow waveform based on 1 of these patients measured by the PC-MRA was used for all cases. The mean flow rate at the ICA was $4 \mathrm{~mL} / \mathrm{s}$.

Flow simulations were conducted using a commercial fluid dynamics simulation package, FLUENT (ANSYS, Lebanon, New Hampshire). The procedure is included in the On-line Appendix. Each case was tested for grid independence in advance and the average mesh had $5 \times 10^{7}$ cells. The detailed simulation procedure is provided in the On-line Appendix.

After simulation, the flow rate through the aneurysm neck $\left(Q_{a}\right)$ was documented, and this flow rate was compared with the flow rate at the ICA $\left(Q_{i}\right)$ to determine the inflow ratio to the aneurysm, $f\left(=Q_{a} / Q_{i}\right)$. The mean value of $f$ over a cardiac cycle was compared with the estimate from high-frame-rate cerebral angiography.

\section{Results}

Fig 2 shows the time curves of the signal for 3 ROIs upstream from the aneurysm and along the parent artery, and these ROIs are $2 \mathrm{~cm}$ apart. The region of interest closest to the aneurysm (CI13) is approximately $1 \mathrm{~cm}$ upstream from the aneurysm. Because of the 2-second injection delay, no signal was seen in the first 2 seconds, and the contrast injection lasts for another 4 seconds before it decreases to the baseline. Quantitative behavior does not change with the selection of different 


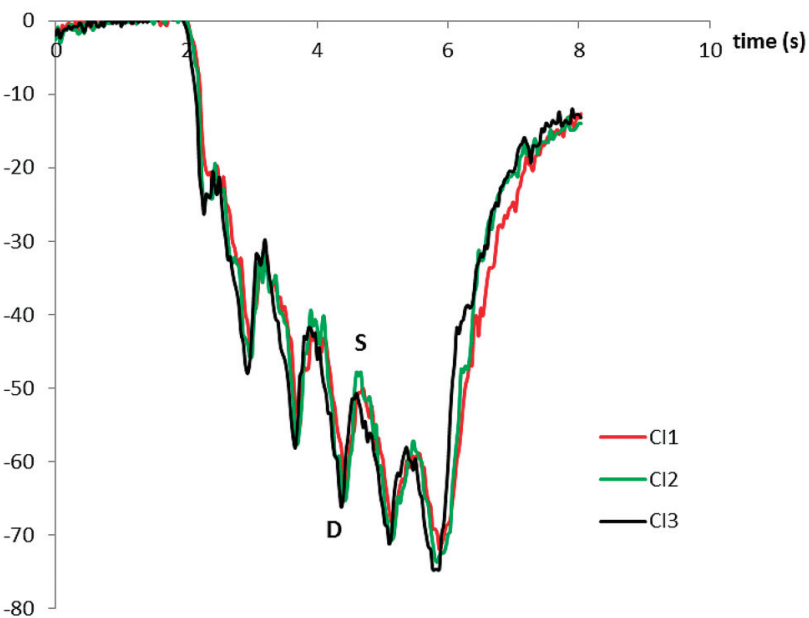

Fig 2. Time courses of $c_{i}$ at 3 different locations that are $2 \mathrm{~cm}$ apart. The unit of $y$-coordinate is $1 / \mathrm{m}$, the same as the attenuation.

regions of interest along the parent vessel, but there is a small time delay in the time curves of these signals. The same dynamic feature is maintained when the region of interest is selected within $5 \mathrm{~cm}$ of the aneurysm and the contrast distribution is not distorted by dispersion or diffusion. ${ }^{13}$ This fact offers a great flexibility in selection of region of interest along the vessel. In Fig 2, D denotes diastole, and $S$ indicates the peak systole. This has been observed previously by Lantz et al. ${ }^{14}$ Their curve is flipped upside down here to conform to our convention that the systole is on the top. At systole, a higher blood flow rate dilutes the contrast injection immediately, so a lower signal is seen at the peak systole. Similarly, contrast concentration is less diluted during diastole and gives a greater signal at diastole. Nevertheless, the magnitude of $c$ represents the contrast concentration despite of the difference in the sign.

Fig 3 demonstrates the flow pattern for case 3 at the peak systole $(A-C)$ and during the diastole $(D-F)$, and streamlines appear to vary with the flow rate. Elevated wall shear stress at $30 \mathrm{~Pa}$ can be seen at the dome at the peak of systole. Fig 3 also shows how the intra-aneurysmal flow is calculated from the sum of all the velocity vectors moving into the aneurysm. The blood leaving the aneurysm is not accounted for because of the incompressibility of the fluid and a constant aneurysm volume. The color of a vector indicates the direction of flow, with the color red for the outflow and the color blue for the inflow. Essentially, the intra-aneurysmal flow ratio is the percentage of arterial blood flow that actually enters the aneurysm. In our CFD calculations, this ratio ranges from $20 \%-100 \%$. The inflow area at the neck apparently does not change during a cardiac cycle and has previously been investigated by Mantha et al. ${ }^{15}$

Dynamic behaviors of $c_{i}, c_{a}$, and $c_{o}$ reveal the intra-aneurysmal hemodynamics in Fig $4 A-C$. These 3 cases, with different $f$ values, are selected for demonstration purposes. An aneurysm of high $f$ produces a $c_{o}$ curve that is very different from $c_{i}$ or $c_{a}$, and all the curves $\left(c_{i}, c_{a}\right.$, and $\left.c_{o}\right)$ are similar for an aneurysm with a small $f$. A high $f$ implies a greater intra-aneurysmal flow and a rapid mixing of the contrast with blood, and the contrast concentration in the aneurysm is often less pulsatile. As a result, the distal contrast concentration loses the signal pulsatility. The contrast dynamic behavior for an aneu-
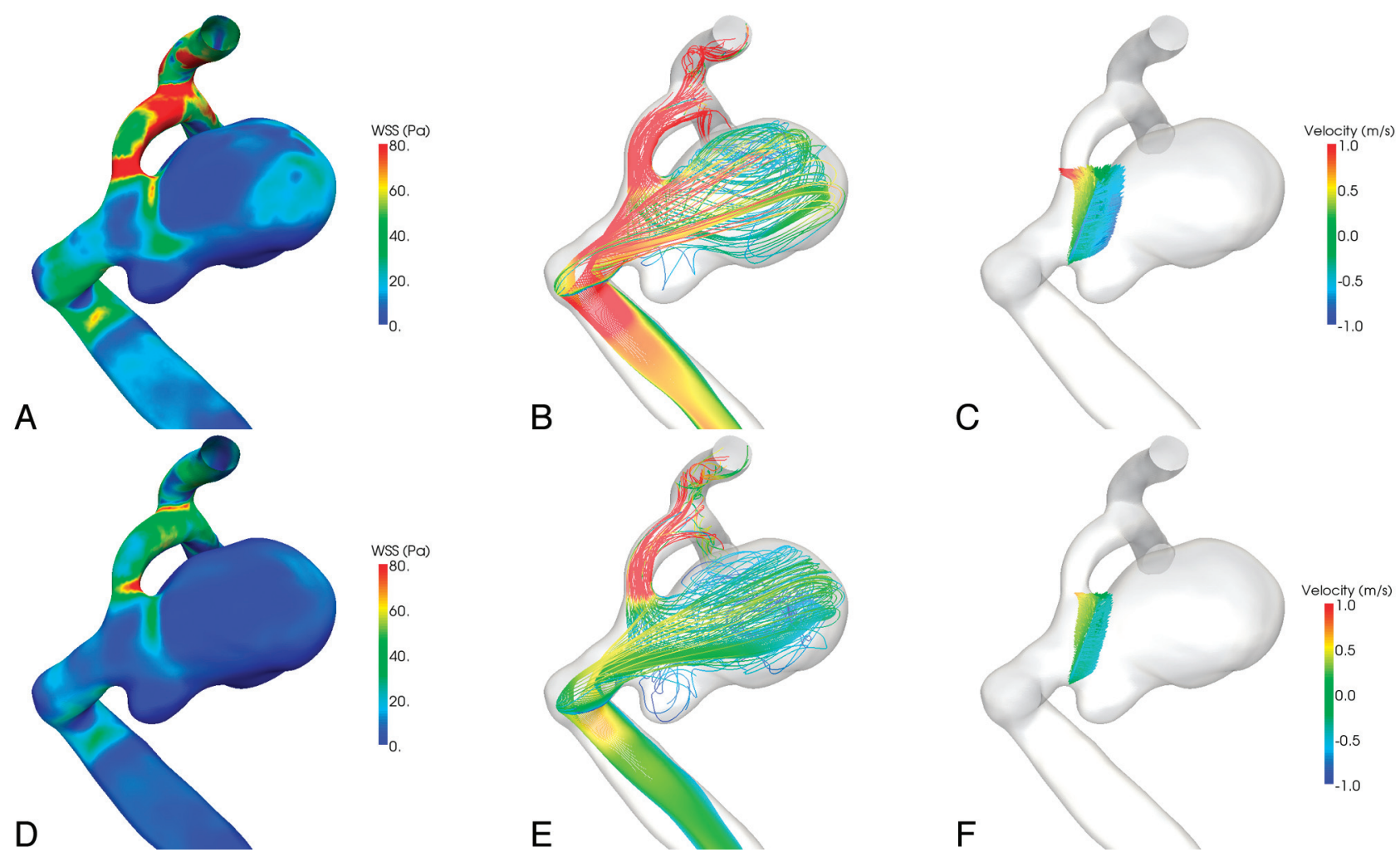

Fig 3. Wall shear stress and streamlines for case 3 at the peak systole $(A-C)$ and during diastole $(D-F)$. $C$ and $F$ are the velocity vectors at the aneurysm neck, and these vectors are used to determine the intra-aneurysmal flow ratio, $f$. 

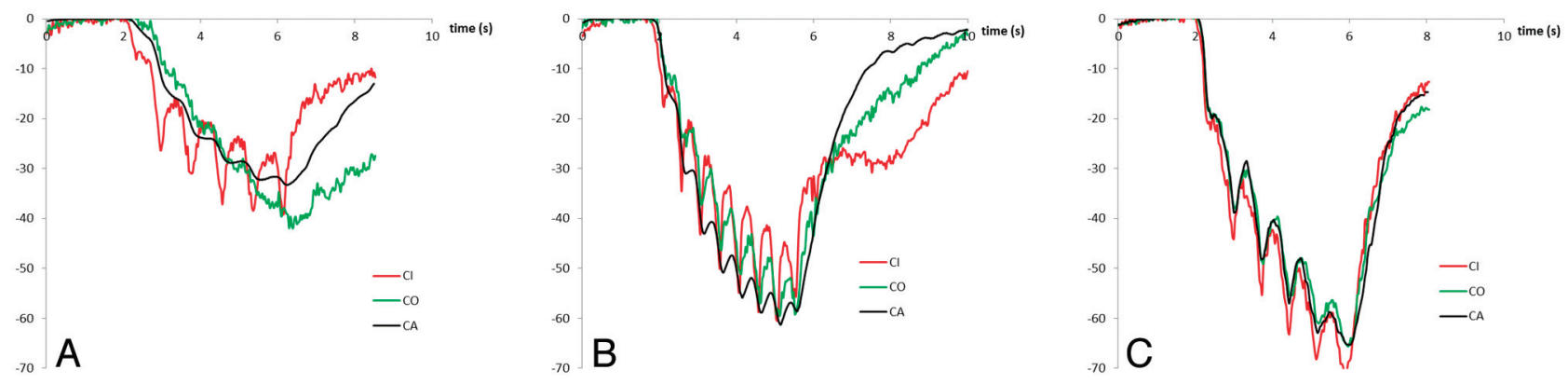

Fig 4. Three examples of $c_{i}, c_{a}$, and $c_{0}$. $A$, Case $1 ; B$, case 3 ; and $C$, case 5 . The intra-aneurysmal flow ratio $(f)$ for these cases is $71 \%$, $56 \%$, and $19 \%$, respectively.
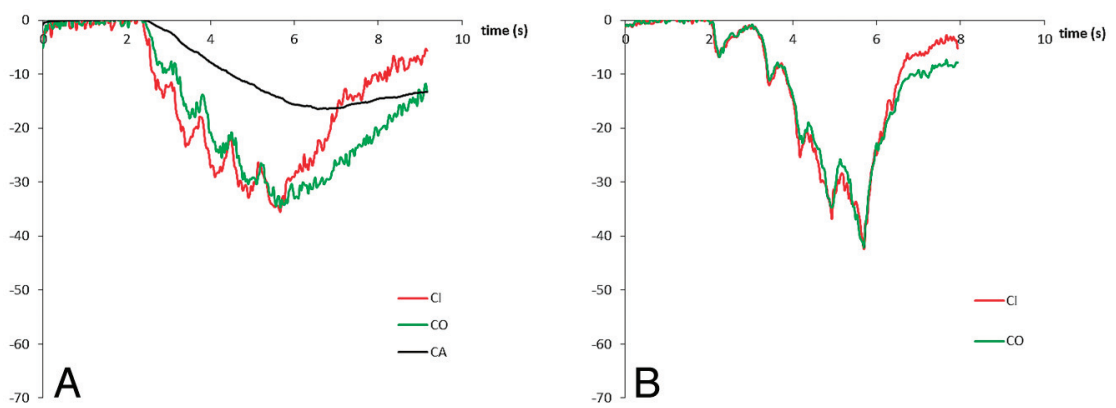

Fig 5. Effect of treatment on $c_{i}$ and $c_{0}$. $A$, Case 1 after flow diverters, and $(B)$ case 5 after coil embolization. For both cases, $f \sim 0 \%$.
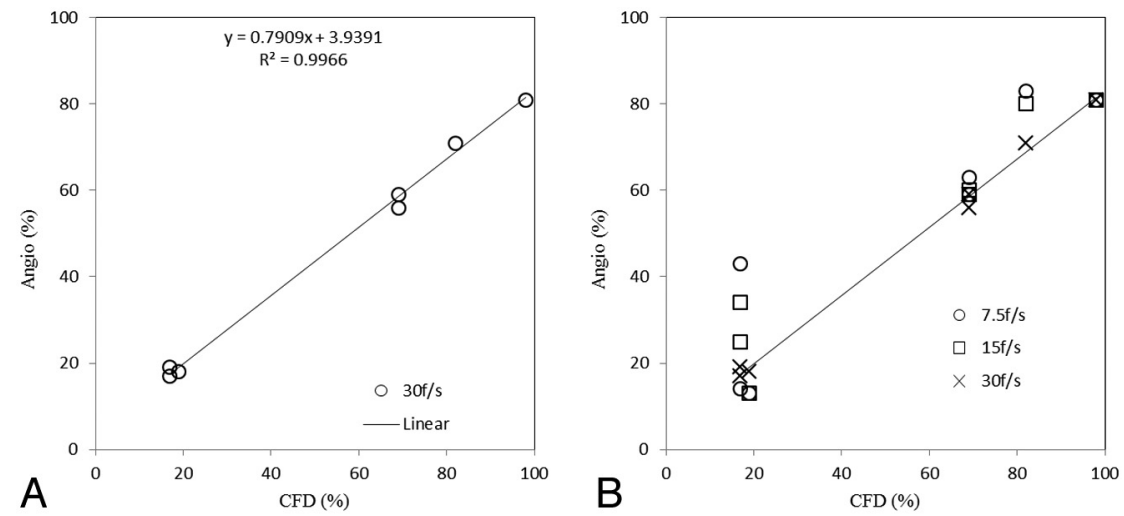

Fig 6. A comparison of aneurysmal inflow ratios obtained from CFD and CA. $A, 30$ frames/s; $B$, down-sampled to lower frame rates (15 frames/s and 7.5 frames/s)

rysm with a low $f$ is dominated by the pulsatility, and pulsatility can be seen in all 3 concentration curves.

Treatment certainly alters the intra-aneurysmal hemodynamics and contrast dynamic behavior. Fig $5 A$ shows the dynamic behavior for the aneurysm in case 1 after 3 Pipeline flow-diverters. The $c_{o}$-curve matches the $c_{i}$-curve much more closely than the $c_{a}$-curve, indicating less flow into the aneurysm and more flow bypassing the aneurysm. The estimate of $f$ based on high-frame-rate cerebral angiography decreases to nearly $0 \%$ from $71 \%$ before the treatment, implying a good flow diversion. Fig $5 B$ presents the $c_{i}$ - and $c_{o}$-curves for case 5 after coil embolization. Because of the presence of coils, the $c_{a}$-curve is not available; nevertheless, agreement between the $c_{i}$-curve and $c_{o}$-curve is improved compared with Fig $4 C$. An estimate of $f$ shows that $f \sim 0 \%$ by assuming a degenerate space for the $c_{a}$. This figure not only shows that the difference due to the treatment is significant but also demonstrates the possibility that these 2 curves $\left(c_{i}\right.$ and $c_{o}$ ) can be used for evaluation of the hemodynamics in intracranial aneurysms in general. This technique allows an immediate assessment of intra-aneurysmal flow during a procedure and provides timely information for interventionalists. In addition, this technique is easy to implement and does not require lengthy calculation or simulation.

The correlation between the CFD calculation and prediction of the intra-aneurysmal flow ratio $f$, based on high-framerate cerebral angiography, is shown in Fig $6 A$. There is a very good correlation between the CFD calculation and our technique, and all data points fall onto a straight line with a slope of $0.79(R=0.99)$; however, our model underestimates the intraaneurysmal flow by $20 \%$. The same 2D DSA images are downsampled to $15 \mathrm{frames} / \mathrm{s}$ and $7.5 \mathrm{frames} / \mathrm{s}$; the analysis is repeated and the results are shown in Fig 6B. Although the linear relationship is intact, the data are scattered farther away from the line, especially for low $f$ cases. Therefore, a high-frame-rate DSA provides a more consistent prediction for the intraaneurysmal flow than the low-frame-rate DSAs do, and 30 frames/s seems to be a reasonable choice. 


\section{Discussion}

We propose a new technique for analysis of intra-aneurysmal flow, and this new technique has been tested on a small number of patients with aneurysm. The intra-aneurysmal flow ratio is found to be proportional to the value predicted by CFD calculation. This new technique offers several advantages over other traditional techniques. First, the new technique shows a beat-to-beat variation of intra-aneurysmal flow that is often ignored in previous analyses. ${ }^{10,11}$ This beat-to-beat variation is especially important for flow-diverter evaluation (Fig 5). Second, the new technique is less affected by the overlap of an aneurysm with its parent vessels. Measuring cross-section area and diameter of a vessel is easier than a similar measurement at an aneurysm. Third, a comparison between signals proximal and distal to the aneurysm provides a unique way of examining the flow activity in the aneurysm. The difference between these 2 signals is independent of the imaging processing because they are acquired at the same run. The $c_{a}$-curves before and after the treatment, however, are subject to different $\mathrm{x}$-ray voltages and electric currents and magnification factors, and a direct comparison of these curves obtained at different angiographic runs may not reveal the same information that we desire. Last, this new technique can serve as a real-time evaluation of aneurysm condition. It can be implemented in a clinical setting and provides an easy and effective way to evaluate flow diversion efficiency.

DSA has been used for flow quantification before, and it has been used by others for velocity measurement, ${ }^{16-18}$ and aneurysmal flow evaluation..$^{9-11}$ These studies demonstrate that the change of signal does have a potential to assist us in understanding the hemodynamics in arteries and aneurysms. The new technique differs from all previous approaches in the location and shape of ROIs, the mathematic model that compares the concentration curves, controlled injection protocol, and the use of 3D DSA. In our experience, it is essential to normalize the signal intensity with the 3D DSA. Normalizing the signal by the area of a region of interest may be convenient, but it does not account for the 3-dimensionality of a complex arterial or aneurysmal geometry. When the vessel is not parallel to the projection plane where the 2D DSA is acquired, comparison between signals at various regions may be a challenge. For example, both a sphere and a cylinder with the same concentration of contrast medium appear on an angiogram as a circle, and normalizing the signal by the area of the circle does not produce a variable that is proportional to the contrast concentration for the sphere. Because 3D DSA provides visualization of anatomy and morphology and is already part of our existing clinical protocol, these 3D images offer us an additional opportunity for better quantifying the signal on $2 \mathrm{D}$ DSA. The detailed mathematic derivation of imaging processing is provided in the On-line Appendix.

Another critical aspect of our technique is the use of a rectangular region of interest rather than a circular region of interest, as is commonly used. If we select a circular region of interest, then the contrast needs to be well mixed with blood; otherwise, the region-of-interest signal will not be representative of the contrast concentration within the bulk flow. To capture both the motion of blood and contrast, the shape of the region of interest of our choice is rectangular and the region of interest includes the entire cross-section of the parent vessel (Fig 1). As long as the contrast moves forward along the vessel, it will appear within the region of interest. At any time interval $d t$, the contrast moving through the region of interest is $Q_{c} d t$, and the blood through the region of interest $Q_{b} d t$, where $Q_{c}$ and $Q_{b}$ are the flow rate of the contrast and blood, respectively. Then the concentration of the contrast is $Q_{c}$ l $\left(Q_{b}+Q_{c}\right)$, and this variable will affect the $\mathrm{x}$-ray signal at the region of interest because the amount of contrast in the region of interest is $V Q_{c} /\left(Q_{b}+Q_{c}\right)$, where $V$ is the volume of the region of interest. This variable is the focus of our measurement. $Q_{c}$ is a constant during the injection $(2 \mathrm{~mL} / \mathrm{s})$, but blood flow $\left(Q_{b}\right)$ is pulsatile, so one cannot expect a constant and steady contrast concentration in the blood at any given time. Because the region of interest covers the entire vessel section, our technique avoids the contrast streaming and other situations where the contrast and blood may not be perfectly mixed. An analogy of our region of interest is a tollbooth on a highway that collects tolls on all the passing contrast and blood. There is no need to require uniform mixing of the contrast and blood, and all we need is the total amounts of contrast and blood passing through the vessel during a short period of time. Whether the contrast is uniformly distributed across the vessel is not required for our technique.

A slow injection rate is required to produce signal pulsatility during the injection. First, the injection does not disturb the blood flow significantly and affect the measurement. Second, $2 \mathrm{~mL} / \mathrm{s}$ is equivalent to half of the mean flow rate at the ICA, so the mean contrast concentration is approximately $33 \%$. At this concentration, the signal can still be treated as linear to the concentration $\left(e^{-x} \sim x\right.$ for small $\left.x\right)$. Signal variation is much smaller once the concentration reaches $80 \%$ because the signal is an exponential function of the attenuation. Dynamic range of digital images limits the range of concentration that we can utilize. In our preliminary in vitro experiments, a faster injection produces mostly saturated or nonpulsatile signals. For example, a $4 \mathrm{~mL} / \mathrm{s}$ injection reduces the signal pulsatility by $70 \%$ and doubles the Reynolds number, which may potentially induce flow instability. For aneurysms in the posterior cerebral circulation, the injection rate may need to be reduced accordingly.

At $125 \mathrm{kV}$, the Hounsfield units for Omnipaque 300 (300 $\mathrm{mgI} / \mathrm{mL}$ ) is $7300,{ }^{19}$ and the linear attenuation coefficient for water is $16 \mathrm{~m}^{-1}, 20$ so the linear attenuation coefficient for Omnipaque 300 is $133 \mathrm{~m}^{-1}$. We can then estimate the blood flow rate using the last few pulses before the end of contrast injection in Fig 4 based on this information; the flow rate for blood is $6.9 \mathrm{~mL} / \mathrm{s}$ for $30 \mathrm{~m}^{-1}, 3.3 \mathrm{~mL} / \mathrm{s}$ for $50 \mathrm{~m}^{-1}$, and 1.9 $\mathrm{mL} / \mathrm{s}$ for $70 \mathrm{~m}^{-1}$. Although the ICA flow rates obtained from attenuation estimate seem to be reasonable and are in the physiologic range, these numbers cannot be verified independently by our technique.

The slope between our prediction and CFD calculation is 0.79 , different from the unity that we anticipated. Several reasons might have contributed to this difference. The attenuation difference between contrast medium and blood is 35\%, and this may produce different flow patterns for contrast medium and for blood, allowing contrast medium residing within the aneurysm for a longer period than blood. In addition, Omnipaque is also more viscous than blood. In CFD simulations, the motion of contrast medium is not explicitly 
modeled, so this difference may not be well appreciated. We assume in our calculation that the contrast and blood are moving independently from each other, so the CFD simulations are conducted based on an arterial flow rate without an injection. The true flow rate after an injection may be very different from the sum of the arterial flow rate and contrast injection rate, and there is currently no proven technique available for an in vivo measurement of this kind. Because the true flow rate with an injection is not available, we opt to use the arterial waveform without an injection. This might introduce a difference in the intra-aneurysmal flow rate. What really interests us is the hemodynamics of an aneurysm at normal condition, not at the time of injection when the patient is under angiographic examination; the purpose of our technique is to establish a correlation between high-frame-rate DSA and the intra-aneurysmal flow at normal condition, so the use of the linear relationship between our technique and CFD calculation provides an easy way to estimate intra-aneurysmal flow. We recognize that the injection of contrast agent may affect the flow field in the artery, but this flow change is difficult to evaluate. The systemic difference of $20 \%$ underestimation by high-framerate DSA can be partly attributed to the effect of injection, but this difference can be easily compensated for each case by the linear coefficient.

Our new technique provides real-time dynamic information on intra-aneurysmal hemodynamics, but it cannot offer wall shear stress distribution on the aneurysm wall. It is also unclear if our technique can be applied to bifurcation aneurysms. The bifurcation itself may affect the signal distribution and this effect is difficult to evaluate based on our experience so far. However, our technique does not have the limitations of most CFD simulations, for which various assumptions need to be made, such as rigid wall, laminar flow, and Newtonian fluid. In vivo $\mathrm{x}$-ray signal behavior captures the true event that occurs inside the aneurysm. Moreover, our analysis is not limited to those aneurysms that have no overlap with other arteries, as the aneurysm signal is not used directly. For cases in which an aneurysm overlaps with arteries, the aneurysmal signal includes a significant contribution from the artery, and an additional adjustment for this arterial signal contribution may be required.

\section{Conclusions}

A new technique has been developed for analysis of intraaneurysmal flow. It was found that there is a linear relationship between intra-aneurysmal flow ratios obtained from cerebral angiography at high frame rate (30 frames/s) and CFD calculation $(R=0.99)$. This linear relationship can be used for real-time assessment of intra-aneurysmal hemodynamics for cerebral aneurysms.

Disclosures: Michel E. Mawad-Research Support (including provision of equipment or materials): Siemens Medical Solutions, Details: Research support for the human and animal facilities; Speakers Bureau: MicroVention, Terumo; Consultant: Codman Neurovascular, Siemens Medical Solutions, Details: Scientific Advisor for both companies.

\section{References}

1. Ferguson GG. Physical factors in the initiation, growth, and rupture of human intracranial saccular aneurysms. J Neurosurg 1972;37:666-77

2. Kulcsar Z, Ugron A, Marosfo IM, et al. Hemodynamics of cerebral aneurysm initiation: the role of wall shear stress and spatial wall shear stress gradient. AJNR Am J Neuroradiol 2011;32:587-94

3. Xiang J, Natarajan SK, Tremmel M, et al. Hemodynamic-morphologic discriminants for intracranial aneurysm rupture. Stroke 2011;42:144-52

4. Augsburger L, Reymond P, Fonck E, et al. Methodologies to assess blood flow in cerebral aneurysms: current state of research and perspectives. J Neuroradiol 2009;36:270-77

5. Cebral JR, Mut F, Weir J, et al. Quantitative characterization of the hemodynamic environment in ruptured and unruptured brain aneurysms. AJNR Am J Neuroradiol 2011;32:145-51

6. Steinman DA, Milner JS, Norley CJ, et al. Image-based computational simulation of flow dynamics in a giant intracranial aneurysm. AJNR Am J Neuroradiol 2003;24:559-66

7. Hope TA, Hope MD, Purcell DD, et al. Evaluation of intracranial stenoses and aneurysms with accelerated 4D flow. Magn Reson Imaging 2010;28:41-46

8. Meckel S, Stalder AF, Santini F, et al. In vivo visualization and analysis of 3-D hemodynamics in cerebral aneurysms with flow-sensitized 4-D MR imaging at 3 T. Neuroradiology 2008;50:473-84

9. Ionita CN, Wang W, Bednarek DR, et al. Assessment of contrast flow modification in aneurysms treated with closed-cell self-deploying asymmetric vascular stents (SAVS). Proc Soc Photo Opt Instrum Eng 2010;7626

10. Sadasivan C, Lieber BB, Cesar L, et al. Angiographic assessment of the performance of flow divertors to treat cerebral aneurysms. Conf Proc IEEE Eng Med Biol Soc 2006;1:3210-13

11. Tenjin H, Asakura F, Nakahara Y, et al. Evaluation of intraaneurysmal blood velocity by time-density curve analysis and digital subtraction angiography. AJNR Am J Neuroradiol 1998;19:1303-07

12. Gockenbach MS. Finite-dimensional linear algebra. Boca Raton, Florida: CRC Press;2010:672

13. Taylor GI. Dispersion of soluble matter in solvent flowing slowly through a tube. Proc Roy Soc A 1953;219:186-203

14. Lantz BM, Foerster JM, Link DP, et al. Determination of relative blood flow in single arteries: new video dilution technique. AJR Am J Roentgenol 1980;134: 1161-68

15. Mantha AR, Benndorf G, Hernandez A, et al. Stability of pulsatile blood flow at the ostium of cerebral aneurysms. J Biomech 2009;42:1081-87

16. Jamison RA, Dubsky S, Siu KK, et al. X-ray velocimetry and haemodynamic forces within a stenosed femoral model at physiological flow rates. Ann Biomed Eng 2011;39:1643-53

17. Rhode KS, Lambrou T, Hawkes DJ, et al. Novel approaches to the measurement of arterial blood flow from dynamic digital X-ray images. IEEE Trans Med Imaging 2005;24:500-13

18. Shpilfoygel SD, Close RA, Valentino DJ, et al. X-ray videodensitometric methods for blood flow and velocity measurement: a critical review of literature. Med Phys 2000;27:2008-23

19. Nyman U, Elmstahl B, Geijer H, et al. Iodine contrast iso-attenuating with diagnostic gadolinium doses in CTA and angiography results in ultra-low iodine doses. A way to avoid both CIN and NSF in azotemic patients? Eur Radiol 2011;21:326-36

20. Hubbell JH. Photon mass attenuation and energy-absorption coefficient from $1 \mathrm{keV}$ to $20 \mathrm{MeV}$. Int J Appl Radiat Isot 1982;33:1269-90 